\title{
Procès-verbal de la séance ordinaire de la Chambre médicale
}

\author{
Jeudi 19 mai 2005, de $9 \mathrm{~h} 30$ à 17h00, Centre de congrès bea bern congress, Berne
}

Annamaria Müller Imboden, secrétaire générale de la FMH

Remarque de la rédactrice du procès-verbal: pour faciliter la lecture, les objets à l'ordre du jour sont numérotés selon la liste et non selon leur ordre de traitement, ce dernier figurant néanmoins entre parenthèses à crochet [ ] .

Début de la séance: 9h40.

\section{Accueil, communications, constitution du bureau}

Jacques de Haller, président de la FMH, souhaite la bienvenue aux personnes présentes, les remercie d'être venues et constate que l'assemblée atteint le quorum nécessaire pour délibérer. Il donne quelques indications d'ordre technique et organisationnel concernant le déroulement de la séance. L'ordre du jour subit quelques modifications en raison de diverses indisponibilités. Jacques de Haller salue en particulier Daniel Lüthi, nouveau responsable du département de la communication en lui donnant l'occasion de se présenter et d'exposer ses idées sur le rôle et l'importance de la communication au sein de la FMH. Le président rend ensuite hommage à Reto Steiner, ancien chargé de presse de la FMH qui a pris sa retraite.

\section{Sont nommés scrutateurs}

Valentin Audétat, Armand Bottani, Giorgio Bugliani, Peter Buser, Robert Cougn, Jörg Fritschi, Jean-Pierre Grob, Niklaus Hasler, Reto Ludwig, Rolf Schlumpf, Jürg Schwegler.

\section{Rapport annuel 2004 de la FMH}

Le rapport statutaire annuel de la FMH, intégré au rapport de gestion, a paru sous forme d'annexe au $\mathrm{n}^{\circ} 18 \mathrm{du}$ Bulletin des médecins suisses du 4 mai 2005. Ce rapport ne suscite aucun commentaire.

La proposition $n^{\circ}$ 2/1 (Comité central) sollicitant l'approbation du rapport annuel est acceptée à l'unanimité sans opposition ni abstention.

\section{Comptes 2004 de la FMH}

Annamaria Müller Imboden, secrétaire générale, présente les principaux résultats de l'exercice financier écoulé. Celui-ci se solde à nouveau par un net excédent des produits pour cause de recettes extraordinaires résultant d'un procès gagné, d'un meilleur rendement de la formation postgraduée et d'une diminution des dépenses dues au recensement de la valeur intrinsèque. Il est prévu d'attribuer de manière rétroactive la part d'excédent de recettes relatives à la formation postgraduée aux réserves de la FPPC. Heribert Riesterer, délégué de l'organe de contrôle PWC, confirme l'exactitude des comptes et recommande de les approuver. Après quelques demandes de renseignement au sujet du procès gagné, du sponsoring et des participations financières, la Chambre passe au vote.

\subsection{Approbation}

La proposition $n^{\circ} 3.1 / 1$ (Comité central)

qui demande d'approuver les comptes annuels 2004 ainsi que les comptes du «Fonds d'assistance» et du «Fonds pour l'encouragement de la formation postgraduée des médecins suisses dans les pays en développement» est acceptée à l'unanimité sans opposition ni abstention. Des remerciements sont exprimés pour la transparence des comptes.

Christoph Ramstein, président du VEDAG, remercie les membres du Comité central et l'ensemble des collaborateurs du Secrétariat général pour leur travail et leur rend hommage au nom des personnes présentes.

\subsection{Décharge au Comité central}

La proposition $n^{\circ}$ 3.2/1 (Comité central) invite la Chambre médicale à suivre les recommandations de l'organe de contrôle. Celle-ci donne à l'unanimité décharge aux membres du Comité central pour l'exercice 2004, sans opposition ni abstention. 


\section{Budget 2006 de la FMH, fixation de la cotisation de membre 2006}

Annamaria Müller Imboden présente le budget de l'année 2006. Elle mentionne à nouveau le passage à la budgétisation stratégique ainsi que les modifications qui en découlent pour la planification, la documentation et la prise de décision. Le budget 2006 est présenté comme équilibré, avec des cotisations de membre restant les mêmes. Les options stratégiques portent sur le maintien de la FPPC, le positionnement du département données, démographie et qualité (DDQ) ainsi que du domaine tarifaire, et sur le développement du département de la communication. En outre, il est prévu d'intensifier la coopération internationale et de mettre un accent sur les contacts nationaux. Des modifications sont également planifiées pour la gestion financière et l'établissement des comptes, sans qu'elles n'aient toutefois d'incidence sur le budget.

Concernant les propositions présentées à la Chambre, la secrétaire générale indique ce qui suit: une proposition de la SVM a été retirée peu avant la séance mais elle figure encore dans les documents; il en va de même pour la contre-proposition du Comité central, également devenue caduque. Pour HIN, il est proposé à la Chambre de reporter ce point à plus tard car le financement des prestations HIN sera examiné durant l'été pour être soumis à la Chambre médicale extraordinaire en décembre. Les deux autres propositions de contribution spéciale seront discutées dans la mesure où les délégués à la Chambre souhaitent leur intégration future dans les cotisations de base. Le Comité central salue cette requête et la prendra en considération dans le budget 2007 .

\section{La proposition $n^{\circ} 4 / 1$ (Comité central)} demandant l'approbation du budget 2006, ainsi que des cotisations des membres figurant ciaprès, est acceptée avec une opposition et sans abstention:

Cotisations de membres 2006

\begin{tabular}{lll} 
Catégorie 1 & Médecins en pratique privée & Fr. 530.- \\
Catégorie 2 & Médecins salariés exerçant une fonction dirigeante & Fr. 530.- \\
Catégorie 3 & $\begin{array}{l}\text { Médecins salariés sans fonction dirigeante et ne se trouvant pas } \\
\text { en formation postgraduée FMH }\end{array}$ & Fr. 265.- \\
Catégorie 4 & Médecins en formation postgraduée & Fr. 265.- \\
\hline Catégorie 5 & Médecins domiciliés et exerçant à l'étranger & Fr. 132.- \\
\hline Catégorie 6 & Membres temporairement sans activité médicale & Fr. 132.-
\end{tabular}

\section{La proposition $n^{\circ}$ 4/2 (Marc Müller, CMPR)} demande de reconduire pour trois années supplémentaires le soutien financier de l'assistanat au cabinet médical des membres de la FMH en formation postgraduée et d'approuver à cet effet une contribution spéciale de Fr. 25.- par membre, valable pour les catégories de cotisation 1, 2, 3 et 4 . Cette contribution est assujettie à l'adoption du budget annuel. Cette proposition est justifiée par le fait que la formation postgraduée en cabinet médical est appréciée et considérée comme indispensable par les intéressés. L'assistanat en cabinet médical est une mesure très importante pour la relève car elle lui donne une approche intéressante de la profession médicale. La probabilité de voir ce projet-pilote devenir une forme régulière de la formation postgraduée augmente sérieusement car le monde politique s'y intéresse de manière accrue. Cette proposition est acceptée avec 1 opposition et 6 abstentions.

\section{La proposition $n^{\circ} \mathbf{4} / 3$ (Comité central)}

sollicite le maintien de la cotisation spéciale de Fr. 20.- par membre, valable pour les catégories de cotisations 1 et 2 pour 2006 et destinée à financer la consolidation nationale des données. La mise à disposition de fonds pour la collecte et la préparation de données n'est pas contestée mais la Chambre se demande toutefois pourquoi ce financement n'est pas intégré dans les coûts ordinaires de la FMH. Le Comité central répond que cette intégration aura lieu à l'achèvement des travaux préliminaires, qui ont besoin de moyens particuliers. La Chambre suit cet argument et accepte cette cotisation avec 2 oppositions et 5 abstentions.

\section{La proposition $n^{\circ} \mathbf{4} / 4$ (Comité central)}

est une proposition inhabituelle, car elle demande de ne traiter le financement des prestations HIN par tous les membres de la FMH qu'à la Chambre médicale extraordinaire du 2 décembre 2005. Après une brève explication donnée par le Comité central sur l'impossibilité de soumettre à la Chambre un projet accompagné d'une proposition sérieuse de financement dans le délai prescrit, les délégués acceptent la proposition sans opposition et avec 5 abstentions. Ils escomptent maintenant que le Comité central leur proposera en fin d'année autre chose qu'une nouvelle demande de prolongation. 


\section{Formation prégraduée, postgraduée et continue (FPPC)}

Max Giger, responsable du domaine de la formation médicale (Medical Education), donne un bref aperçu de la situation actuelle et des développements en cours dans le domaine de la formation postgraduée et continue. Les points centraux sont constitués par l'accréditation, la création de titres fédéraux de spécialiste et les travaux concernant la loi sur les professions médicales (LPMéd). Le Département fédéral concerné ne partage pas toujours l'avis de la FMH mais celleci a le ferme espoir de parvenir à des solutions constructives dans les différents dossiers.

\subsection{Création d'un titre de spécialiste en chirurgie de la main}

La proposition $n^{\circ}$ 5.1/1 (Comité central) demande la création d'un titre de spécialiste en chirurgie de la main afin de soutenir les efforts entrepris pour une chirurgie de la main compétitive, de haute qualité et quantitativement bien représentée. La CFPC est favorable à la création de ce titre, mais pas toutes les sociétés concernées. Les critiques concernent avant tout le manque de compatibilité avec l'UE, les doublons possibles dans les programmes de formation postgraduée et les problèmes liés à la garantie des droits acquis (valeur intrinsèque). On se demande également quelles pourraient être les conséquences en cas de rejet par le chef du département concerné. Le Comité central demande de ne pas placer les questions tarifaires avant la qualité qui est primordiale. La compétence de créer un titre fédéral relève clairement de la Confédération et la FMH ne tient pas à s'engager sur deux voies (elle ne créera pas de titres FMH en parallèle aux titres fédéraux). En considération des points de vue exprimés, la Chambre accepte la proposition avec 9 oppositions et 19 abstentions.

\subsection{Commission pour la formation postgraduée et continue (CFPC)}

\section{a) Siège de la Société suisse des médecins homéopathes (SSMH)}

La proposition $n^{\circ}$ 5.2.a/1 (Bruno Ferroni, SSMH) sollicite le droit, pour la Société suisse des médecins homéopathes (SSMH), de siéger à la Commission pour la formation postgraduée et continue (CFPC). Les conditions sont données; en vertu de l'article $41,4^{\mathrm{e}}$ alinéa des statuts de la FMH, les sociétés médicales assumant la responsabilité d'une formation approfondie ou d'une attestation de formation complémentaire peuvent siéger à la CFPC. Cette proposition est acceptée par 76 voix contre 47 et 21 abstentions.

\section{b) Siège de la Société médicale suisse de thérapie neurale (thérapie de régulation) selon Huneke (SMSTN)}

La proposition $n^{\circ}$ 5.2.b/1 (Andreas Beck, SMSTN) est analogue à la proposition $n^{\circ} 5.2$.a/ 1 et sollicite, pour la Société médicale suisse de thérapie neurale (thérapie de régulation) selon Huneke (SMSTN), le droit de siéger à la Commission pour la formation postgraduée et continue (CFPC). Cette proposition est également acceptée par 76 voix contre 47 et 28 abstentions.

\section{Activités politiques}

Le Conseiller national Yves Guisan, vice-président de la FMH, donne un aperçu des délibérations parlementaires importantes pour la FMH. Il s'agit concrètement des points suivants:

- La procédure de consultation relative au blocage de l'accès à la pratique privée (ordonnance fédérale du 3 juillet 2002 sur la limitation de l'admission des fournisseurs de prestations à pratiquer à la charge de l'assurancemaladie obligatoire): au sujet du blocage de l'accès à la pratique privée, la FMH a été reçue en audience, a exprimé sa désapprobation de principe, a exposé les points critiques et en a appelé à la responsabilité de la Confédération concernant les effets possibles de ce blocage sur la couverture en soins médicaux.

- La LPMéd: Lors des délibérations relatives à la LPMéd, la FMH a appris avec plaisir qu'une seule organisation compétente en la matière est prévue pour la formation postgraduée. La solidarité avec d'autres organisations professionnelles et les universités est tout aussi réjouissante. Par contre, le rejet du «droit à la formation postgraduée» n'est pas satisfaisant. L'assurance responsabilité civile professionnelle obligatoire menace de devenir un problème aigu même si on ne le remarque pas encore, car les médecins éprouvent des difficultés accrues à conclure des polices d'assurance financièrement supportables. De nombreux assureurs classent le corps médical comme groupe à haut risque avec des clauses contractuelles en conséquence.

- La révision de la LAMal et l'orientation prise: La Commission du Conseil des Etats va traiter la question du financement hospitalier et celle de la liberté de contracter. 
De manière générale, la FMH renforce et professionnalise ses activités de prise d'influence au Parlement.

Après la discussion consacrée à ce point de l'ordre du jour, la Chambre exprime ses vifs remerciements à la FMH pour son engagement tout en regrettant de voir les demandes du corps médical apparemment peu prises en compte. Les sociétés cantonales sont invitées à documenter de façon précise la pénurie de médecins et à en informer les autorités cantonales. Au bout du compte, l'application du blocage de l'accès à la pratique privée relève de la compétence des cantons.

\section{La proposition $n^{\circ}$ 6/1 (Hans Kurt, FMPP)}

invitant le Comité central à tout mettre en œuvre pour que le blocage de l'accès à la pratique privée soit supprimé ou du moins que le problème relatif au travail à temps partiel et à l'interruption de l'activité médicale soit examiné et résolu, est transmise sous forme de mandat au Comité central qui l'accepte volontiers.

\section{Tarifs et contrats}

Franco Muggli, responsable du domaine tarifaire et du groupe de projet Tarifs du Comité central, donne quelques informations sur le TARMED, non sans remercier auparavant cordialement toutes les personnes impliquées. Dans les travaux et lors des négociations, on s'est rendu compte combien il est important de disposer de ses propres données, d'autant plus que le tarif, qui relevait à l'origine de la gestion d'entreprise, a passé dans le domaine de l'économie publique et que la menace d'un budget global pour les soins ambulatoires n'est pas loin. Bien que les paramètres et instruments de mesure à la base des critères EAE n'aient pas encore été finalisés, la pression politique en vue d'un contrôle des coûts par une mécanique tarifaire reste entière. A l'aide d'exemples éclairants, Franco Muggli montre que le fait «d'avoir des données» n'est de loin pas suffisant et que les résultats doivent être interprétés avec grand soin car ils présentent toujours une image nuancée quand on les observe de plus près. Avec le projet «NAKO», on est en train de mettre en place et de consolider une gestion des données. C'est d'autant plus important que les «conventions TARMED» subséquentes sont en voie d'élaboration et de conclusion. Franco Muggli note que les négociations et les procédures décisionnelles sont longues et complexes dans les organes tarifaires; il compte sur la compréhension de chacun si les conventions des sociétés médicales doivent subir en partie des re- tards importants et des pertes de contenu. Les négociations tarifaires ne peuvent pas, en effet, être assimilées à des commandes de livraison.

Res Haefeli, président sortant du G7, présente l'état des lieux concernant la réglementation qui suivra la neutralité des coûts. Un mois avant la fin de la phase de la neutralité des coûts, et donc avant la fixation des valeurs définitives du point tarifaire, les travaux vont bon train. Différents points restent à clarifier et à mettre au net, entre autres les incohérences de la banque de données de santésuisse. Un point délicat concerne le traitement du volume compensatoire mesuré d'après les volumes «trop utilisés» ou «non épuisés» des cantons et des régions de valeur du point tarifaire, volume résultant de la différence par rapport à la valeur du point tarifaire neutre en termes de coûts constatée de manière rétroactive. Quelques cantons doivent rétrocéder des montants énormes, d'autres auraient encore des volumes non utilisés. Les valeurs intermédiaires et définitives seront également déterminées à l'aide des volumes de correction. Il est prévu de discuter de tous ces problèmes lors d'une conférence des présidents qui suivra la Chambre médicale et de décider la suite concrète à y donner. A cet effet, il s'agit en particulier de la question cruciale concernant l'harmonisation de la valeur du point tarifaire. Une fois la présentation de Res Haefeli terminée, ce dernier point donne lieu à d'âpres discussions. Le principe n'est pas contesté mais les points de vue s'opposent quant à la voie à suivre et au calendrier à respecter. Des critiques sont également émises concernant l'absence du domaine ambulatoire hospitalier. Ici, la FMH ne peut rien entreprendre, car la compétence de négocier est aux mains de l'association hospitalière. Pour terminer, Res Haefeli donne encore des informations concernant le projet de convention sur les prestations et les coûts, destiné à remplacer la convention sur la neutralité des coûts. Contrairement à cette dernière, la nouvelle convention ne contiendra toutefois aucune disposition contraignante, mais seulement des recommandations aux sociétés cantonales de médecine qui récupéreront la direction des négociations à la fin de la phase de neutralité des coûts. L'objectif visé est de créer des règles si possible uniformes et partenariales pour parvenir au but, c'est-à-dire pour déterminer les valeurs du point tarifaire et éviter les organes de recours. Cependant, il n'est pas possible d'échapper à l'influence du préposé à la surveillance des prix qui doit obligatoirement être consulté par les gouvernements cantonaux dans le cadre de la procédure d'approbation et/ou de fixation de la valeur du point. De même, la convergence sou- 
haitée s'avère problématique si les conventions sont appliquées exclusivement au niveau cantonal et de manière séparée, pour la pratique en cabinet privé et les soins ambulatoires à l'hôpital. Voilà la situation sur le plan légal. Après une discussion animée, Res Haefeli est très cordialement remercié pour tout le travail accompli.

Franco Muggli présente rapidement la situation dans le domaine LAA. Ici, la phase consacrée à la stabilité des coûts est achevée depuis longtemps et la valeur du point tarifaire est fixée de manière définitive. Soutenue par l'OFSP, la CTM fait maintenant pression pour la conclusion d'une convention contenant un nouveau pilotage des coûts de caractère permanent. Néanmoins, le fait est qu'il existe des conventions en vigueur non dénoncées et que la Confédération ne dispose d'aucun pouvoir décisionnel au niveau juridique.

Olivier Kappeler, membre du Comité central chargé du domaine de la valeur intrinsèque, informe sur l'état actuel de la banque de données de la valeur intrinsèque, dont la mise en service est maintenant prévue pour le second semestre de l'année. Le traitement des affaires en suspens avance conformément au calendrier et devrait s'achever dans les délais prévus, pour autant que les médecins concernés y participent de manière constructive et rapide. En parallèle, la FMH a déposé une demande auprès de la Commission de la concurrence en accord avec santésuisse. Cette demande est composée de deux questions: le droit des cartels est-il applicable dans le domaine de la valeur intrinsèque? Une approbation en vertu du droit fédéral remplace-t-elle, le cas échéant, les bases légales manquantes? La demande a fait l'objet d'un accusé de réception mais la FMH attend encore la réponse.

\section{La proposition $n^{\circ}$ 7/1 (Jürg Schlup, Berne)} demande au Comité central ce qu'il a l'intention de faire lorsque la convention tarifaire pour les prestations paramédicales au cabinet médical aura pris fin et ce qu'il conseillera aux membres de la FMH. La situation juridique est tout sauf claire. Hanspeter Kuhn, secrétaire général adjoint de la $\mathrm{FMH}$, indique qu'il existe déjà une jurisprudence du Tribunal fédéral concernant le personnel engagé au cabinet médical. Le Comité central estime qu'il sera possible de trouver prochainement une solution commune avec santésuisse. Le proposant déclare être satisfait de cette information.

\section{La proposition $n^{\circ}$ 7/2 (Charles Selleger, Genève)}

demande au Comité central de parvenir, dans les négociations avec les assureurs opérant en tiers payant (LAMal et LAA), à ce que les paiements aux médecins soient effectués au moyen du système BVR lorsque le médecin l'utilise pour ses factures (bulletins de versement orange avec numéro de référence). A l'heure où l'informatisation des cabinets médicaux a été rendue obligatoire, il est illogique que les assureurs n'adoptent pas le système BVR, qui simplifie la gestion électronique des données au cabinet médical. La proposition est acceptée sans vote par le Comité central en tant que mandat de négociation.

La proposition $n^{\circ}$ 7/3 (Beat Manser, Lucerne) vise à remettre au G7 le mandat de négocier une répartition symétrique du volume de compensation. Il est choquant que les cantons dotés d'un très bas niveau de coûts au début de la phase de neutralité des coûts, puis d'un niveau moyen restant au-dessous de la moyenne suisse, soient «punis» par des valeurs du point tarifaire peu élevées. La proposition est retirée pour des raisons formelles car la Chambre médicale n'est pas légitimée à remettre des mandats de négociation aux sociétés cantonales. La proposition est toutefois transmise à la Conférence des présidents cantonaux ayant lieu le même jour pour y être discutée.

\section{Code de déontologie}

[traité après le point 11]

\subsection{Approbation de directives de I'ASSM}

Jusqu'à présent, il n'existait qu'une seule directive pour la prise en charge des patients en fin de vie. Comme cette directive ne tenait pas suffisamment compte de la situation pratique, Ursula Steiner-König, vice-présidente de la FMH, explique qu'on a procédé à des différenciations qui ont abouti à quatre directives en fonction de différents contextes. La formation des médecins concernés n'est certes pas indiquée de manière explicite mais elle est implicitement contenue dans les directives.

\section{La proposition $n^{\circ} 8.1 / 1$ (Comité central)} demandant que les quatre directives médicoéthiques:

- Questions des limites de la médecine intensive;

- Traitement et prise en charge de patients de longue durée avec un dommage cérébral important;

- Traitement et prise en charge de patients âgés nécessitant des soins;

- Traitement et prise en charge de patients en fin de vie 
de l'Académie suisse des sciences médicales (ASSM) pour la prise en charge des patientes et patients en fin de vie soient insérées dans le code de déontologie de la FMH est acceptée à la majorité avec une opposition et 4 abstentions.

\section{Projet HOP-Line}

[traité après le point 7]

Des hôtes sont présents à la Chambre médicale pour ce point de l'ordre du jour: Dr Christiane Margairaz, du conseil interdisciplinaire pour la médecine et la prévention de la violence (CIMPV), hôpital universitaire de Genève et Dr Judit Pòk, médecin-chef adjointe à la policlinique des femmes de l'hôpital universitaire de Zurich.

En introduction, Ursula Steiner-König, viceprésidente de la FMH, rappelle la décision de la Chambre médicale du 11 octobre 2003, qui a chargé le Comité central de traiter le problème de l'inconduite sexuelle dans le secteur professionnel (Professional Sexual Misconduct PSM) et de soumettre un projet concret à la Chambre médicale ordinaire 2005. Pour ce faire, le Comité central a mis sur pied un groupe de travail en vue de la réalisation du mandat sous la direction de Werner Tschan. Ce groupe a présenté son rapport en automne 2004 et l'a clos pour sa transmission à la Chambre médicale en le complétant avec 17 recommandations. Ces derniers temps, les médias ont rapporté différents incidents, parfois de façon tapageuse, en lien avec la PSM et ont indiqué que la Chambre de ce jour en discuterait. Au nom du Comité central, Ursula Steiner-König précise à ce sujet que la nouvelle LPMéd prévoit que l'autorité cantonale de surveillance peut ordonner des mesures disciplinaires. Aujourd'hui déjà, les commissions de déontologie ont donné au corps médical des possibilités de sanctions qui vont jusqu'à la publication dans les journaux spécialisés des sociétés médicales, y compris la FMH, communication à l'autorité concernée et aux assureurs incluse. Comme la Suisse au contraire des Etats-Unis par exemple - ne connaît aucun devoir d'annoncer des médecins coupables de PSM, le nombre colporté par les médias d'environ 14000 cas par année n'est pas crédible et encore moins prouvé. Cette rumeur médiatique est regrettable et projette une lumière défavorable sur un sujet sensible et douloureux.

Dans sa présentation, Christiane Margairaz explique les conséquences des abus perpétrés par des médecins à l'encontre de patients: la personne malade devient encore plus malade, ce qui contredit le postulat de guérison ancré dans le code de déontologie, d'une part, et détruit la relation de confiance, d'autre part, et donc la condition de base par excellence de l'acte médical. En outre, l'ensemble du corps médical acquiert une mauvaise réputation. L'objectif est donc d'attirer l'attention des jeunes médecins sur le fait qu'ils ne doivent jamais utiliser leur «position de force» pour satisfaire leurs propres besoins et qu'ils ne doivent pas chercher la sexualité et l'érotisme dans la relation médecinpatient.

Judit Pòk résume le contenu du rapport du groupe de travail. Tout en se focalisant sur la PSM, ce rapport est toutefois valable pour toutes les formes d'inconduite professionnelle. En outre, il est clairement orienté sur une offre de soutien pour les médecins - d'où le nom de «HOP», abréviation de Help Our Physicians - sachant qu'avec une telle approche, on vient aussi implicitement en aide aux patients. Le rapport et ses recommandations portent essentiellement sur le conseil individuel. L'offre proposée dans la dernière recommandation de la liste consiste en une ligne téléphonique, un site internet et un regroupement de spécialistes à disposition pour le conseil et le soutien. Le regroupement de spécialistes devrait encore être créé et les personnes formées à cet effet. Judit Pòk indique toutefois que le groupe de travail n'exige pas impérativement le maintien des recommandations mais qu'il estime essentiel que la FMH traite sérieusement ce problème, qu'elle se distancie clairement de l'inconduite professionnelle en son sein comme à l'extérieur et qu'elle prenne des mesures concrètes pour la prévention et l'aide nécessaire.

Le rapport et les recommandations du groupe de travail donnent lieu à des appréciations diverses dans la discussion qui suit; les données quantitatives et qualitatives ne peuvent, en particulier, pas être suivies. Les orateurs sont unanimes à dire qu'aucun degré de tolérance ne doit être accordé à la PSM et que le sujet doit être traité avec sérieux. La proposition du groupe de travail pour une ligne téléphonique fait problème car on ignore si les gestionnaires de la ligne n'encourent pas des sanctions pénales en annonçant aux autorités les cas dont ils ont pris connaissance. Néanmoins, les abus sexuels sont des délits répréhensibles. En outre, les délégués se demandent s'il est judicieux d'investir pendant plusieurs années des sommes importantes dans un projet avant d'avoir effectué des investigations pour savoir si les médecins impliqués dans une PSM téléphoneront ou enverront des e-mails et quel en sera le nombre par année. Au service de conseils de Bâle, personne ne s'est annoncé jusqu'à présent. 


\section{La proposition $n^{\circ}$ 9/1 (Comité central)}

est présentée par René Raggenbass, co-responsable du domaine «Santé et prévention». Celui-ci constate que le Comité central condamne totalement les abus sexuels dans la relation médecinpatient, en estimant que les propositions du groupe de travail HOP-Line ne sont pas des mesures adéquates pour résoudre le problème (ligne de téléphone aboutissant au secrétariat général? Site internet?) et que le rapport avec la réalité suisse n'a pas été examiné. Pour cette raison, le Comité central propose à la Chambre médicale de renvoyer le projet au groupe de travail avec mandat de concevoir un projet-pilote limité à trois ans, réalisable avec un budget annuel d'environ Fr. $10000 .-$. Le projet-pilote devra contenir des propositions concrètes de mesures aptes à contrer la PSM; ces mesures devront être proches de la réalité et avoir fait l'objet de tests.

\section{Vote d'entrée en matière}

Tout d'abord, la Chambre se prononce sur la question de savoir s'il faut poursuivre l'étude du dossier consacré à l'inconduite professionnelle. Les délégués se prononcent clairement en faveur d'un suivi par 3 oppositions et 8 abstentions.

Le vote suivant oppose le projet HOP-Line à la contre-proposition du Comité central, cette dernière l'emporte sans opposition et avec 7 abstentions. La proposition du Comité central est donc clairement approuvée.

\section{Numéro d'appel urgent valable dans toute la Suisse}

[traité après le point 8]

Marc Müller, président du forum du sauvetage de la FMH, informe sur les délibérations et recommandations du forum en ce qui concerne le numéro d'appel urgent (à trois chiffres) valable dans toute la Suisse: en 1996 déjà, dans ses 12 thèses sur le sauvetage et publiées dans le Bulletin des médecins suisses [1], la FMH avait de-

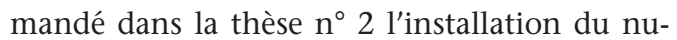
méro d'appel d'urgence sanitaire 144 sur tout le territoire suisse, exonéré de taxe et comme point de contact pour toutes les situations médicales d'urgence. Le forum considère que ce postulat est approprié aujourd'hui encore et recommande pour cette raison de renoncer à son propre numéro à trois chiffres pour le service d'urgence médicale, car il en résulterait des confusions. Le forum est en train d'élaborer des bases décisionnelles contenant un aperçu des différentes formes d'organisation du service médical d'urgence en Suisse, à l'attention des sociétés cantonales, des gouvernements cantonaux auxquels revient la compétence d'organiser le service d'urgence et de la CDS. L'IAS a donné son accord de participation.

\section{Conceptions du corps médical suisse en matière de qualité}

[traité après le point 9]

En introduction, Christoph Ramstein, co-président du VEDAG, explique rapidement l'arrièreplan de la proposition mise en discussion. HansAnton Vogel, membre du VEDAG, présente les «Lignes directrices du corps médical suisse en matière de qualité», soumises le 21 avril 2005 aux présidentes et présidents des organisations représentées à la Chambre médicale et publiées dans le Bulletin des médecins suisses [2]. L'initiateur tient à souligner qu'il ne s'agit pas d'une affaire interne au VEDAG, que le groupe de travail proposé est ouvert, qu'il doit se composer de représentants de toute la Suisse et qu'on tiendra compte des connaissances en la matière en y intégrant des experts du Secrétariat général.

\section{La proposition $n^{\circ}$ 11/1 (Christoph Ramstein, VEDAG)}

demande la formation d'un "groupe de travail Qualité FMH», chargé du mandat suivant:

a. développer en permanence les lignes directrices;

b. encourager la mise en œuvre de leur contenu;

c. élaborer un concept pour un forum de la qualité, pour la formation des personnes chargées du domaine de la qualité et pour la mise en place de contrôles de qualité;

d. tamer des négociations avec les interlocuteurs concernés sur la base des conceptions du corps médical suisse en matière de qualité.

La proposition est acceptée avec 5 oppositions et 9 abstentions.

\section{HIN: collaboration, projets}

Ce point est repoussé à la Chambre médicale du 2 décembre 2005 .

\section{Divers}

Aucune communication.

Le président de la FMH clôt la séance à 16h35. 